\title{
SILÍCIO E CLORETO DE SÓDIO NA NUTRIÇÃO MINERAL E PRODUÇÃO DE MATÉRIA SECA DE PLANTAS DE MORINGA (Moringa oleifera LAM.) ${ }^{(1)}$
}

\author{
J . R. P. MIRANDA(2), J . G. CARVAL HO(3), D. R. SANTOS ${ }^{(4)}$, A. L. O. \\ FREIRE ${ }^{(4)}$, J . C. BERTONI(5), J . R. M. MELO ${ }^{(6)}$ \& A. L. CALDAS ${ }^{(6)}$
}

\begin{abstract}
RESUMO
Realizou-se um experimento em casa de vegetação do Departamento de Ciência do Solo da Universidade Federal de Lavras, para avaliar o efeito do silício na nutrição mineral e na produção de matéria seca de plantas de moringa submetidas ao estresse salino. Utilizaram-se o esquema fatorial e o delineamento de blocos casualizados, com três repetições, e uma planta por vaso de $3 \mathrm{~L}$ de capacidade, em que o primeiro fator referiu-se às doses de $\mathrm{NaCl}(0,30,60,90$ e $\left.120 \mathrm{~mol} \mathrm{~m}^{-3}\right)$ e o segundo às doses de $\mathrm{SiO}_{2}\left(0,0,5,1,0\right.$ e 1,5 $\left.\mathrm{mol} \mathrm{m}^{-3}\right)$, em solução de Hoagland \& Arnon (1950) a $50 \%$ da concentração normal. As soluções foram renovadas em intervalos de 10 dias e, aos 35 dias, após a aplicação dos tratamentos, as plantas foram colhidas, separadas em folhas, caule e raízes, secas, para determinação da produção de matéria seca, e moídas, para determinação dos teores de N, P, K, Ca, Mg, S, Na, Cl e Si. A partir dos teores, determinaram-se as relações $\mathrm{Na} / \mathrm{K}, \mathrm{Na} / \mathrm{Ca}$ e $\mathrm{Na} / \mathrm{Mg}$ e a translocação dos nutrientes para a parte aérea. Observou-se que a adição do Si elevou os teores de $\mathrm{K}$ e de $\mathrm{Ca}$ nas folhas e reduzi u os de $\mathrm{Na}$ e as relações $\mathrm{Na} / \mathrm{K}, \mathrm{Na} / \mathrm{Ca}$ e $\mathrm{Na} / \mathrm{Mg}$. O Si não reduziu os efeitos depressivos do $\mathrm{NaCl}$ na produção de matéria seca das plantas de moringa.
\end{abstract}

Termos de indexação: solução nutritiva, estresse salino, silício.

(1) Parte da Tese de Doutorado do primeiro autor, apresentada ao Curso de Pós-Graduação em Solos e Nutrição de Plantas da Universidade Federal de Lavras - UFLA, para a obtenção do título de Doutor em Solos e Nutrição de Plantas. Recebido para publicação em abril de 2000 e aprovado em setembro de 2002.

(2) Professor do Departamento de Engenharia Florestal, Universidade Federal de Campina Grande - UFCG. Caixa Postal 64, CEP 58700-000 Patos (PB). E-mail:paesr@bol.com.br

(3) Professora Titular do Departamento de Ciência do Solo, Universidade Federal de Lavras - UFLA. Caixa Postal 37, CEP 37200000 Lavras (MG). E-mail: jguedes@ufla.br

(4) Professores do Departamento de Engenharia Florestal, UFCG. E-mails: diercules@ul.com.br; lofreire@cstr.ufpb.br

(5) Doutorando do Departamento de Ciência do Solo, UFLA. E-mail: julio bertoni@cargil.com

(6) Estudante de Graduação, CSTR/UFCG. 
SUMMARY: SILICON AND SODIUM CHLORIDE EFFECTS ON MINERAL NUTRITION AND DRY MATTER PRODUCTION OF MORINGA (Moringa ol eifera LAM.) PLANTS

\begin{abstract}
A greenhouseexperiment was conducted in theSoil ScienceDepartment of theF ederal University of Lavras, Brazil, to evaluate the effect of silicon on mineral nutrition and dry matter production of moringa plants, cultivated under salinity stress. The experiments followed a randomized factorial block-type design, in three replications. In pots of $3 \mathrm{~L}$ capacity with one plant each, five levels of $\mathrm{NaCl}\left(0,30,60,90\right.$, and $\left.120 \mathrm{~mol} \mathrm{~m}^{-3}\right)$ were combined with four levels of $\mathrm{SiO}_{2}\left(0.0,0.5,1.0\right.$, and $\left.1.5 \mathrm{~mol} \mathrm{~m}^{-3}\right)$ in Hoagl and and Arnon nutrient solution at $50 \%$ of the normal concentration. The sol utions were renewed every 10 days and the plants harvested after 45 days. Leaves, stems, and roots were separately dried for the determination of dry matter and elemental contents - N, P, K, Ca, Mg, S, Na, $\mathrm{Cl}$, and $\mathrm{Si}$. Therelations $\mathrm{Na} / \mathrm{K}, \mathrm{Na} / \mathrm{Ca}$, and $\mathrm{Na} / \mathrm{Mg}$ were determined. $\mathrm{K}$ and $\mathrm{Ca}$ contents increased while the $\mathrm{Na}$ content and the $\mathrm{Na} / \mathrm{K}, \mathrm{Na} / \mathrm{Ca}$, and $\mathrm{Na} / \mathrm{Mg}$ relations in the leaves decreased under the influence of silicon. Silicon did not reduce the restrictive effects of $\mathrm{NaCl}$ on the dry matter production of the moringa plants.
\end{abstract}

Index terms: nutritive solution, salinity stress, silicon.

\section{INTRODUÇÃO}

O desenvolvimento de técnicas que permitam a integração de áreas degradadas pela salinidade ou pela exploração ao sistema produtivo, utilizando espécies florestais nativas ou exóticas, como a moringa (Moringa oleifera Lam.), poderá elevar a oferta de produtos e reduzir a pressão de consumo sobre a vegetação nativa. Esta espécie, embora exótica, pode ser encontrada em diversas regiões do semi-árido brasileiro, tolera o estresse hídrico e, segundo Miranda \& Carvalho (1998), é halofílica. Pode ser utilizada na alimentação humana eanimal (Cáceres et al., 1991), na produção de óleo (J ahn, 1992) como planta medicinal (Peter, 1979; Morton, 1991) e na clarificação de água para o consumo humano, característica bastanterel evante, tendo em vista que diversas localidades do semi-árido brasileiro ainda não dispõem do tratamento adequado de água.

Apesar do exposto, o cultivo desta espécie é insignificante, em virtude, principalmente, da falta de conhecimento quanto às suas exigências nutricionais. Estudos sobre o seu comportamento em relação à salinidade são importantes, uma vez que sua área abrange as regiões mais secas do País e de maior ocorrência de solos salinos. O emprego do silício para reduzir o estresse salino sobre seu crescimento poderá transformar-se numa alternativa viável aos métodos tradi cionais de recuperação euso de sol os afetados por sais.

De acordo com J effrey \& Izquierdo (1989), os efeitos imediatos da salinidade sobre os vegetais podem ser resumidos em seca fisiológica, provocada pela redução no potencial osmótico, pelo desbalanço nutricional, considerando a el evada concentração iônica, a inibição, pelo sódio, da absorção de outros cátions e o efeito tóxico de íons sódio e cloro.

A tolerância ao estresse sal ino pode ser resultante do controlena aquisiçãoe ealocação de Na pela planta, do ajustamento osmótico (Cheeseman, 1988), da compartimentalização do $\mathrm{Na}$, da presença de pequenas porções do citoplasma com alta mobilidade, envolvidas no transporte, distribuição e compartimentalização dos íons (J escke \& Wolf, 1988), além do acúmulo de compostos nitrogenados, como mecanismo de manutenção da atividade metabólica vegetal (Broetto, 1995).

Estima-se que, apenas no semi-árido brasileiro, a área ocupada por solos naturalmente salinos é superior a nove milhões de hectares, predominando no interior do Polígono das Secas. Em termos mundiais, a perda de terras devida à salinização secundária é superior às áreas incorporadas ao sistema produtivo pel o desmatamento (Frommer et al., 1999). De modo geral, a pesquisa científica tem contribuído para a redução dos efeitos da sal inidade sobre as plantas por meio da avaliação de métodos de recuperação de solos e da identificação de mecanismos que, mediante o melhoramento genético, conferem às plantas tolerância à salinidade (Broetto, 1995).

Para corrigir a salinidade dos sol os, promove-se, normalmente, a lixiviação dos sais através do seu perfil mediante a aplicação de condicionadores. No entanto, esta prática pode ser pouco eficiente em regiões que não disponham de água em qualidadee quantidade adequadas. Alternativamente, a aplicação de Si como amenizador do estresse sal ino parece viável e decorre dos resultados obtidos por 
Bradbury \& Ahmad (1990), com algaroba (Prosopis juliflora); por Liang et al. (1996), com cevada, e por Matoh et al. (1986), com arroz. Os autores supracitados demonstraram que o Si promove elevação da produção de matéria seca e assimilação de $\mathrm{CO}_{2}$, aumentos da relação $\mathrm{K} / \mathrm{Na}$ e dos teores foliares de $\mathrm{K}$ em plantas sensíveis e tolerantes. Portanto, admite-se que os solos afetados por sais, notadamente aquel es cuja recuperação por métodos tradicionais seja inviável, econômica ou tecnicamente, possam ser utilizados em exploração agrossilvopastoril, adotando-se técnicas alternativas e espécies de múltiplo uso.

Objetivou-se avaliar, neste trabalho, o efeito amenizador e tóxico do Si e do $\mathrm{NaCl}$, respectivamente, sobre a produção de matéria seca da parte aérea e raízes e sobre os teores foliares de $\mathrm{N}, \mathrm{P}, \mathrm{K}$, $\mathrm{Ca}, \mathrm{Mg}, \mathrm{S}, \mathrm{Na}, \mathrm{Cl}$ e $\mathrm{SiO}_{2}$ em plantas de moringa, cultivadas em solução nutritiva.

\section{MATERIAL E MÉTODOS}

Realizou-se um experimento em casa de vegetação do Departamento de Ciência do Solo da Universidade Federal de Lavras, entre junho e agosto de 1999. Sementes de moringa (Moringa oleifera Lam.) foram semeadas em ver mi culita, cuja umidade foi mantida com água destilada. Após 15 dias, as plantas foram transferidas para vasos plásticos com $3 \mathrm{~L}$ de capacidade que continham solução de Hoagland \& Arnon (1950) a $50 \%$ da concentração, à qual foram aplicados o $\mathrm{SiO}_{2}$ $\left(\mathrm{Na}_{2} \mathrm{SiO}_{3}\right)$ e o $\mathrm{NaCl}$.

$\mathrm{O}$ delineamento experimental adotado foi em blocos ao acaso, de um fatorial $5 \times 4$, com três repetições e uma planta por vaso. Os fatores referiram-se às concentrações de $\mathrm{NaCl}(0 ; 30 ; 60 ; 90$; e $120 \mathrm{~mol} \mathrm{~m}^{-3}$ ) e de $\mathrm{SiO}_{2}\left(0 ; 0,5 ; 1,0\right.$; e 1,5 $\left.\mathrm{mol} \mathrm{m}^{-3}\right)$, respectivamente. As soluções foram renovadas em interval os de 10 dias, coletando-se as plantas 35 dias após a aplicação dos tratamentos. As plantas foram separadas em fol has, caul e eraízes e levadas à estufa com circulação forçada de ar a 70 C, para secagem até peso constante. Posteriormente, determinou-se a produção de matéria seca e procedeu-se à moagem do material para fins de análises químicas. No extrato obtido por digestão nítrico-perclórica, foram determinados: os teores de $\mathrm{P}$, por colorimetria; Ca e Mg, por espectrofotometria de absorção atômica; K e $\mathrm{Na}$, por fotometria de emissão em chama, eS, por turbidimetria do sulfato de bário (Malavol ta et al., 1997). Os teores de $\mathrm{N}$ total foram determinados pelo método semimicro Kjeldahl (Liao, 1981), sendo a destilação ea titulação efetuadas segundo Bremner \& Edwards (1965). Os teores de $\mathrm{Cl}$ foram determinados por titulometria do nitrato de prata (Malavolta et al., 1997) e os de Si pelo método colorimétrico do "azul-de-molibdênio (Furlani \& Galo, 1978).
As variáveis estudadas foram submetidas às análises de variância e de regressão, cujas equações foram ajustadas às médias, considerando os fatores isolados ou a interação desses fatores.

\section{RESULTADOS E DISCUSSÃO}

\section{Teores foliares de macronutrientes, $\mathrm{Na}, \mathrm{Cl}$ e $\mathrm{SiO}_{2}$ e as relações $\mathrm{Na} / \mathrm{K}, \mathrm{Na} / \mathrm{Ca}$ e $\mathrm{Na} / \mathrm{Mg}$}

Independentemente do teor de $\mathrm{SiO}_{2}$ na solução, o $\mathrm{NaCl}$ exerceu efeito quadrático sobre os teores de $\mathrm{N}$, cujo teor máximo ocorreu com $37,9 \mathrm{~mol} \mathrm{~m}^{-3}$ de $\mathrm{NaCl}$ (Figura 1a) e posterior decréscimo acentuado com o aumento de sua concentração. Efeito semel hante foi observado por Saur et al. (1995), em plantas de pinus, e por Miranda (2000), em plantas de cajueiro, sensíveis à salinidade, embora, no cajueiro, o teor máximo tenha ocorrido na dose de $\mathrm{NaCl}$ mais el evada $\left(97,3 \mathrm{~mol} \mathrm{~m}^{-3}\right.$ de $\left.\mathrm{NaCl}\right)$. O efeito positivo depende do tempo de exposição das plantas ao estresse salino, das suas diferenças genotípicas quanto à tolerância à salinidade (Perez-Alfocea, 1993) e da fonte de $\mathrm{N}$ empregada (Botella et al., 1997). A adição de $\mathrm{NaCl}$ inibe a absorção de $\mathrm{NO}_{3}{ }^{-}$ provavelmente, em virtude do efeito depressivo do $\mathrm{Cl}^{-}$, mas não exerce efeito sobre a absorção de $\mathrm{NH}_{4}{ }^{+}$, embora não tenha sido comprovada eventual competi ção entre el es durante o processo de absorção (Botella et al., 1994).

Os teores de $\mathrm{P}$ foram influenciados pela interação do $\mathrm{NaCl}$ e o $\mathrm{SiO}_{2}$. No entanto, apenas os dados obtidos com 0,5 mol m-3 de $\mathrm{SiO}_{2}$ apresentaram ajuste quadrático significativo, com valor máximo em $85,7 \mathrm{~mol} \mathrm{~m}^{-3}$ de $\mathrm{NaCl}$ (Figura $1 \mathrm{~b}$ ). Tal comportamento deveu-se, provavel mente, à redução na atividade do íon fosfato e em conseqüência de sua absorção e transporte para as fol has, provocada pelo aumento da concentração de $\mathrm{NaCl}$ na solução de cultivo, conforme ressaltam Al-Karaki (1997) e Awad et al. (1990).

A interação do $\mathrm{NaCl}$ eo $\mathrm{SiO}_{2}$ influenciou os teores de $K$. Os dados ajustaram-se a regressões quadráticas, com decréscimos significativos (Figura 2a). Observou-se que os teores obtidos com $0,0,0,5$ e $1,0 \mathrm{~mol} \mathrm{~m}^{-3}$ de $\mathrm{SiO}_{2}$ apresentaram comportamento semel hante, embora deva ser ressal tado que, na presença do $\mathrm{SiO}_{2}$, os valores foram sempre mais elevados do que na sua ausência. De modo geral, a aplicação do $\mathrm{SiO}_{2}$ foi favorável à absorção etransporte deK para as fol has, causados, provavel mente, pel o aumento da permeabilidade da membrana ao K, e pela redução do $\mathrm{Na}$, conforme observaram Liang et al. (1996) em plantas de cevada.

O aumento da sel etividade da membrana ao K é de fundamental importância para reduzir os efeitos da salinidade sobre as plantas, visto tratar-se do 
principal componente do ajustamento osmótico (Reggiani et al., 1995), do qual dependem a manutenção do turgor das células e a continuidade dos processos de divisão e crescimento celulares (Hsiao et al., 1976), além da produção vegetal em ambiente salino (Yeo, 1983).

À semel hança do que ocorreu com os teores de K, os de Ca também foram influenciados pela interação do Si e $\mathrm{NaCl}$. Na ausência do Si ou mediante a aplicação de $0,5,1,0$ ou $1,5 \mathrm{~mol} \mathrm{~m}^{-3} \mathrm{SiO}_{2}$, os teores de $\mathrm{Ca}$ decresceram com o aumento da concentração de $\mathrm{NaCl}$, segundo um model o quadrático (Figura 2b). De modo geral, os menores teores de Ca foram obtidos em plantas tratadas apenas com $\mathrm{NaCl}$, mostrando que a aplicação do Si foi favorável ao processo de absorção e transporte do nutriente para as fol has. Observou-se, ainda, que, na concentração de $60 \mathrm{~mol} \mathrm{~m}^{-3}$ de $\mathrm{NaCl}$, a aplicação de $0,5 \mathrm{~mol} \mathrm{~m}^{-3}$ de
$\mathrm{SiO}_{2}$ propiciou maiores teores de $\mathrm{Ca}$ nas folhas, podendo ter provocado inibição na absorção do K, enquanto, nas concentrações superiores, os teores foram mais baixos e pouco diferenciados, independentemente da concentração de $\mathrm{SiO}_{2}$ na solução.

Esta tendência está de acordo com os dados obtidos por Lynch \& Läuchli (1995), segundo os quais a redução na absorção de Ca parece ser controlada por mecanismos fisiológicos, e que o $\mathrm{NaCl}$, além de inibir o movimento na raiz deste nutriente em direção ao xilema, pode reduzir sua disponibilidade na solução, graças à precipitação e à formação de pares iônicos.

Houve redução drástica dos teores foliares de Mg, com a el evação da concentração de $\mathrm{NaCl}$ e de $\mathrm{SiO}_{2}$ na solução (Figuras 3a,b). Em ambos os casos, os
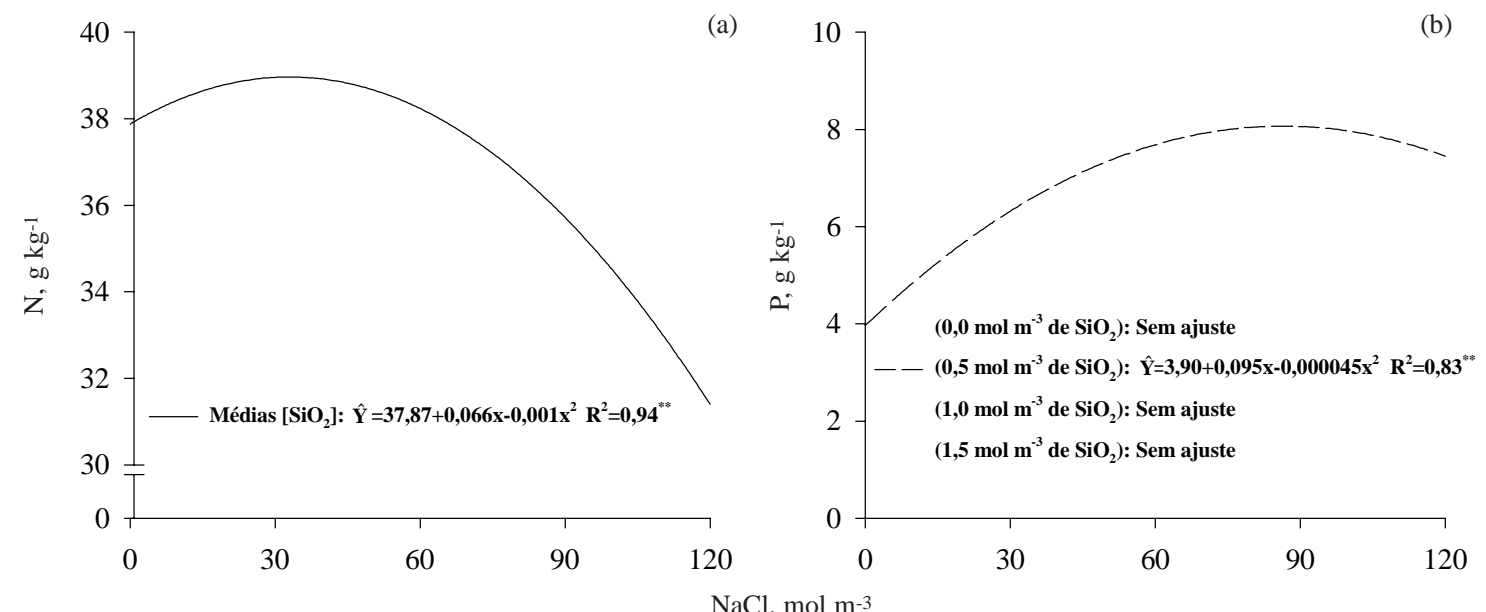

Figura 1. Teores de N (a) e P (b) nas folhas de moringa, considerando a concentração de $\mathrm{NaCl}$, e de $\mathrm{NaCl}$ e $\mathrm{SiO}_{2}$ na solução, respectivamente.
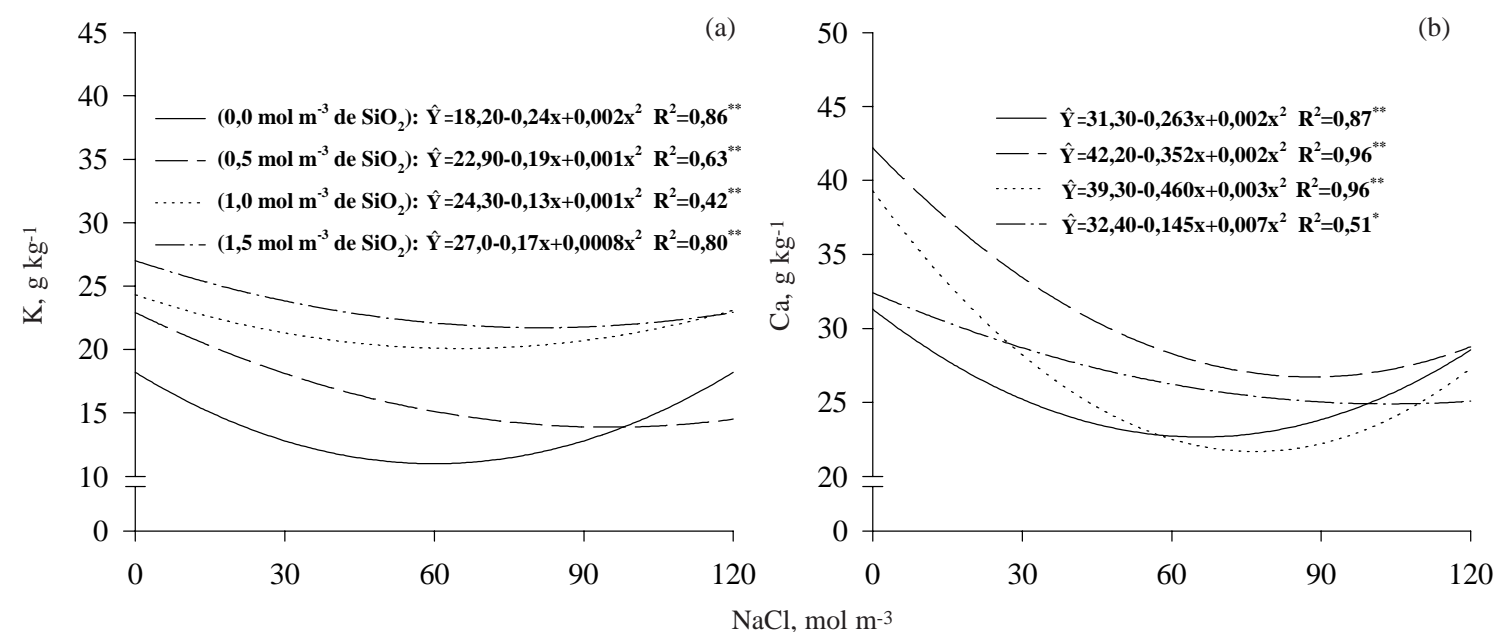

Figura 2. Teores de $\mathrm{K}$ (a) e $\mathrm{Ca}$ (b) nas fol has de moringa, considerando a concentração de $\mathrm{NaCl}$ e de $\mathrm{SiO}_{2}$ na solução. 
dados apresentaram ajustamento quadrático, podendo-se observar que, inicialmente, o Si favoreceu a absorção do nutriente. Efeito semel hante foi observado por Miranda (2000), em plantas de cajueiro tolerantes à salinidade, aplicando $1,0 \mathrm{~mol} \mathrm{~m}^{-3}$ de $\mathrm{SiO}_{2}$, e por Liang et al. (1996), em plantas de cevada.

De modo geral, observou-se que os teores de $K$, Ca e Mg (Figuras 2a,b e 3a) tenderam a ser mais altos nas maiores doses de $\mathrm{NaCl}$, em relaçãoàs doses intermediárias, independentemente da aplicação ou não de Si. Isto pode ter sido causado pelo efeito de concentração, uma vez que, nestas condições, as plantas apresentaram os menores acúmulos de matéria seca.
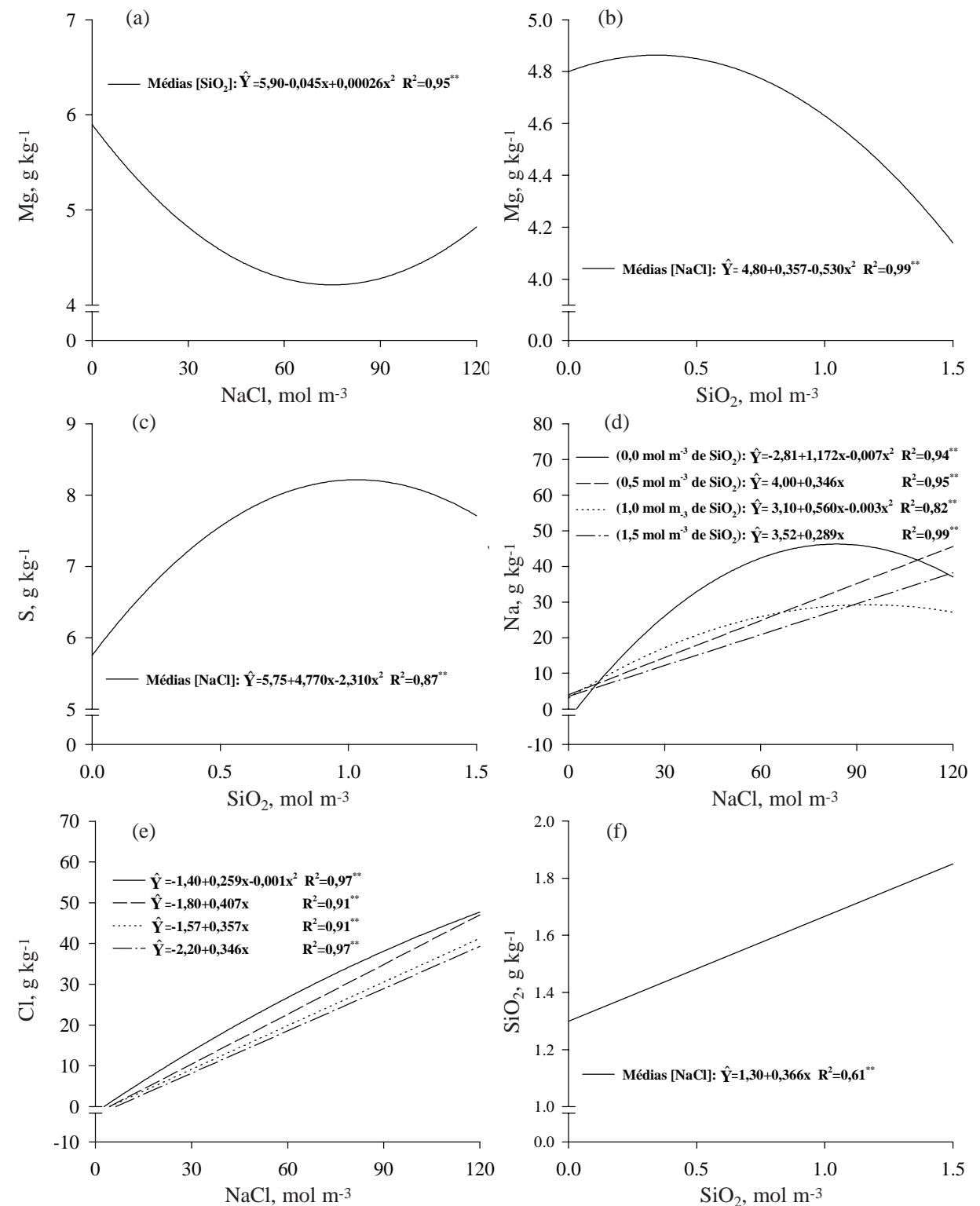

Figura 3. Teores de $\mathrm{Mg}(\mathrm{a}, \mathrm{b}), \mathrm{S}(\mathrm{c}), \mathrm{Na}(\mathrm{d}), \mathrm{Cl}(\mathrm{e}) \mathrm{e} \mathrm{SiO}_{2}$ (f) nas folhas de moringa, considerando as concentrações de $\mathrm{NaCl}$ e de $\mathrm{SiO}_{2}$ na solução.
Os teores de S sofreram influência apenas do Si, ajustaram-se à regressão quadrática (Figura $3 c$ ) e aumentaram com a concentração de $\mathrm{SiO}_{2}$ na sol ução. Estes resultados concordam com os encontrados por Liang et al. (1996), que atribuíram o aumento na absorção de nutrientes à maior seletividade da membrana promovida pelo Si.

Houve interação dos fatores em estudo e os teores foliares de $\mathrm{Na}$ (Figura $3 \mathrm{~d}$ ). As plantas que não receberam $\mathrm{Si}$ tenderam a apresentar teores mais elevados de $\mathrm{Na}$ nas fol has, com ajuste quadrático dos dados, semelhante ao que ocorreu quando se aplicou $1,0 \mathrm{~mol} \mathrm{~m}^{-3}$ de $\mathrm{SiO}_{2}$. Nas demais doses de $\mathrm{Si}$, os teores de $\mathrm{Na}$ aumentaram linearmente com o $\mathrm{NaCl}$. O fato de as plantas crescidas em soluções 
que continham Si terem apresentado menores teores de $\mathrm{Na}$ converge para as observações feitas por Liang et al. (1996), devendo-se, provavel mente, à redução da permeabilidade da membrana ao $\mathrm{Na}$.

$\mathrm{O} \mathrm{Cl}$ apresentou comportamento semel hante ao $\mathrm{Na}$. De modo geral, seus teores aumentaram com o $\mathrm{NaCl}$ e diminuíram com o $\mathrm{SiO}_{2}$ na sol ução nutritiva. Os teores foliares de $\mathrm{Cl}$, embora crescentes com o $\mathrm{NaCl}$, foram inferiores a $50 \mathrm{~g} \mathrm{~kg}^{-1}$, limiteconsiderado tóxico para as plantas sensíveis, sendo, portanto, insuficientes para inibir a síntese protéica (Frota \& Tucker, 1978).

A redução na concentração de $\mathrm{Na}$ e, principalmente, de $\mathrm{Cl}$, pelo $\mathrm{Si}$, embora tenha sido discreta, não pode deixar de ser considerada, pois grande parte das conseqüências da salinidade está relacionada com a nutrição catiônica, principal mente com a redução na absorção de K e Ca (Bernstein \& Ayres, 1953; Geraldson, 1957; Kramer et al., 1977), em razão do acúmulo excessivo de $\mathrm{Na}$ e $\mathrm{Cl}$ (Marschner, 1995), resultando na diminuição do crescimento vegetal.

Os teores de Si não foram influenciados pelo $\mathrm{NaCl}$, mas aumentaram linearmente com a concentração de $\mathrm{SiO}_{2}$ na solução (Figura 3f). I sto parece ser importante, pois, conforme foi observado por Bradbury \& Ahmad (1990) e Liang et al. (1996), o aumentona concentração de $\mathrm{SiO}_{2}$ no meio de cultivo, bem como o conseqüente aumento na sua absorção, favoreceu diversas características analisadas em plantas de algaroba e de cevada. Todavia, é necessário considerar que, no presente trabalho, pode ter ocorrido efeito de concentração nos teores de $\mathrm{SiO}_{2}$ e deS, em decorrência da influência negativa do $\mathrm{SiO}_{2}$ na produção de matéria seca das fol has.

As relações $\mathrm{Na} / \mathrm{K}, \mathrm{Na} / \mathrm{Ca}$ e $\mathrm{Na} / \mathrm{Mg}$, representadas pela razão entre os teores desses el ementos na planta, apresentaram comportamento semel hante. De modo geral, os maiores valores ocorreram nas plantas que receberam apenas o $\mathrm{NaCl}$, com ajuste quadrático dos dados (Figura 4a,b,c). Os dados referentes à relação $\mathrm{Na} / \mathrm{K}$, obtidos mediante a aplicação de $1,0 \mathrm{~mol} \mathrm{~m}^{-3}$ de $\mathrm{SiO}_{2}$, nãoapresentaram ajuste matemático, mas aumentaram linearmente adicionando-se 0,5 e 1,5 mol m${ }^{-3} \mathrm{deSiO}_{2}$. As relações $\mathrm{Na} / \mathrm{Ca}$ e $\mathrm{Na} / \mathrm{Mg}$, apresentaram resposta quadrática ao $\mathrm{NaCl}$ ao serem aplicados 1,5 e $1,0 \mathrm{~mol} \mathrm{~m}^{-3} \mathrm{deSiO}_{2}$, respectivamente, enquanto com 0,5 e $1,5 \mathrm{~mol} \mathrm{~m}^{-3} \mathrm{de}$ $\mathrm{SiO}_{2}$ aumentaram linearmente com o estresse sal ino. Deve-se salientar que as plantas que cresceram na presença de $\mathrm{SiO}_{2}$ apresentaram relações $\mathrm{Na} / \mathrm{K}, \mathrm{Na} /$ $\mathrm{Ca}$ e $\mathrm{Na/Mg}$ inferiores àquel as que cresceram na sua ausência, evidenciando o efeito benéfico deste elemento na absorção dos macronutrientes catiônicos em detrimento do $\mathrm{Na}$.
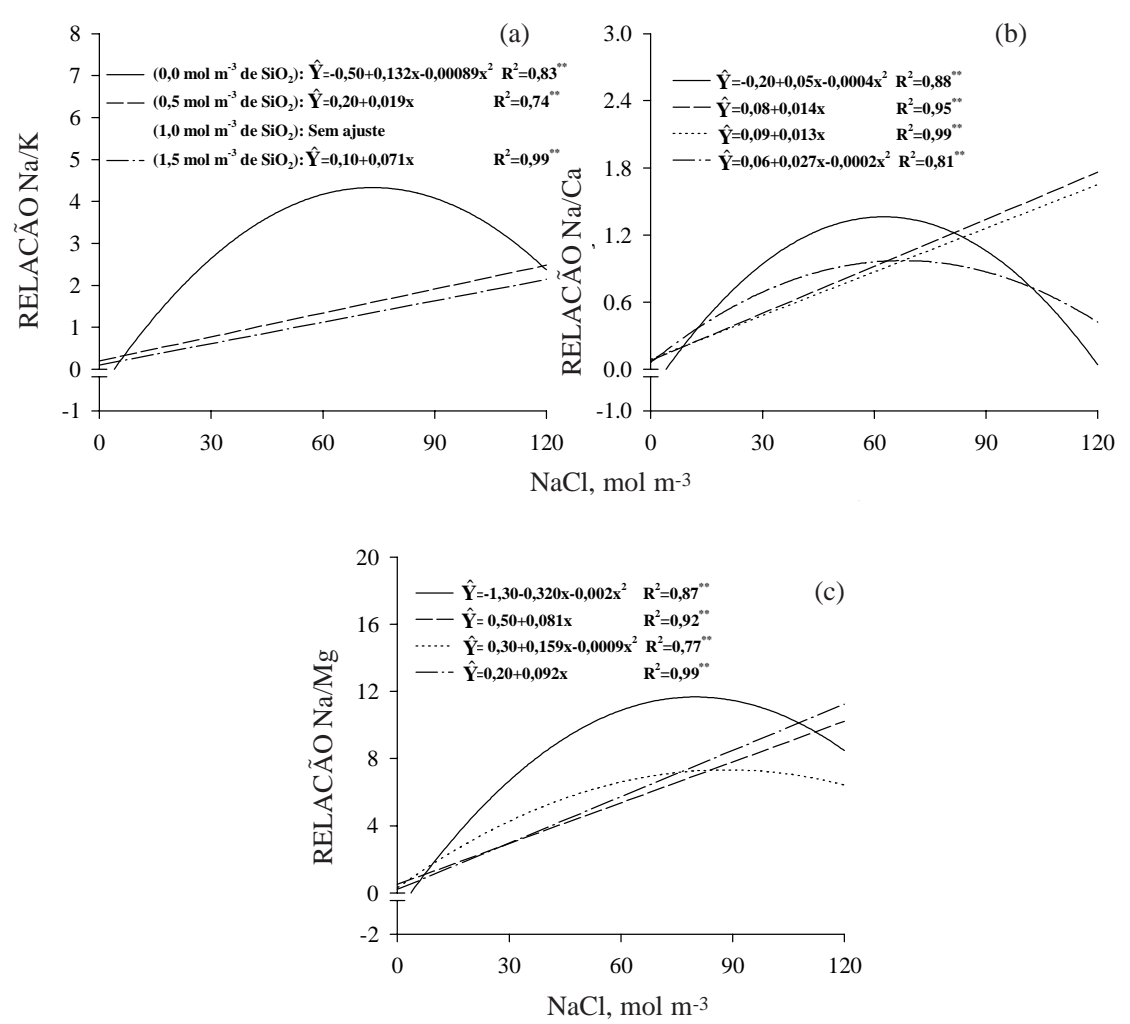

Figura 4. Relações $\mathrm{Na} / \mathrm{K}$ (a), $\mathrm{Na} / \mathrm{Ca}$ (b) e $\mathrm{Na} / \mathrm{Mg}$ (c), considerando a concentração de $\mathrm{NaCl}$ e de $\mathrm{SiO}_{2}$ na solução. 
A avaliação dessas relações é importante por indicar a habilidade das plantas em absorver sel etivamente os macronutrientes catiônicos, especialmente $\mathrm{K}$ eCa, quando submetidas a estresse salino, graças à influência destes na regulação estomática, nas trocas gasosas, na manutenção da integridade das membranas e, em conseqüência, no metabolismo vegetal. De acordo com Greenway \& Munns (1980), as plantas tolerantes apresentam baixas relações $\mathrm{Na} / \mathrm{K}$ no floema e no citoplasma e elevadas no vacúolo, em decorrência da baixa sel etividade do tonoplasto ao $\mathrm{Na}$.

\section{Acúmulo de matéria seca nas folhas, caule, raízes e total}

A interação do $\mathrm{NaCl}$ e $\mathrm{SiO}_{2}$ foi significativa quanto à produção de matéria seca de fol has, caule e total (Figura 5a,b,d). Na ausência do Si ou mediante a aplicação de $0,5 \mathrm{~mol} \mathrm{~m}^{-3}$ de $\mathrm{SiO}_{2}$, a produção de matéria seca das fol has apresentou uma redução quadrática, enquanto, nas demais concentrações, a redução foi linear (Figura 5a). Comportamento semel hante foi observado para o caule e produção total, quando se consideraram a ausência do Si ou a adição de $1,0 \mathrm{~mol} \mathrm{~m}^{-3}$ de $\mathrm{SiO}_{2}$ (Figura 5b,d). Nas demais concentrações de $\mathrm{SiO}_{2}, \mathrm{O}$ acúmulo de matéria seca pelo caule e o total decresceram linearmente. A produção de matéria seca das raízes foi influenciada apenas pelo $\mathrm{NaCl}$, decrescendo linearmente com o aumento das concentrações do sal na sol ução nutritiva (Figura 5c).

De modo geral, os dados estão de acordo com Yeo et al. (1985), atribuindo-se o efeito mais comum da sal inidade sobre o crescimento vegetal à redução da área foliar e, conseqüentemente, à fotossíntese. $\mathrm{O}$ Si não foi capaz de amenizar os efeitos depressivos do $\mathrm{NaCl}$ na produção de matéria seca das plantas de moringa, conforme haviam observado Matoh et al. (1986), Bradbury \& Ahmad (1990) e Liang et al . (1996).

A tolerância das plantas ao excesso de sais envolve, além do crescimento celular, a manutenção do transporte de íons durante o crescimento e o equilíbrio entre o potencial osmótico e a expansão celular (Zimmerman, 1978). Elevados teores de sais perturbam este equilíbrio nas plantas glicófitas e, em conseqüência, reduzem substancialmente o crescimento celular, embora as taxas de transporte dosíons mantenham adequadoturgor. Deacordocom Benzil et al . (1989), não se podeexduir a possibilidade deser a restrição à expansão cel ular um ajustamento necessário em ambientes ricos em sais.
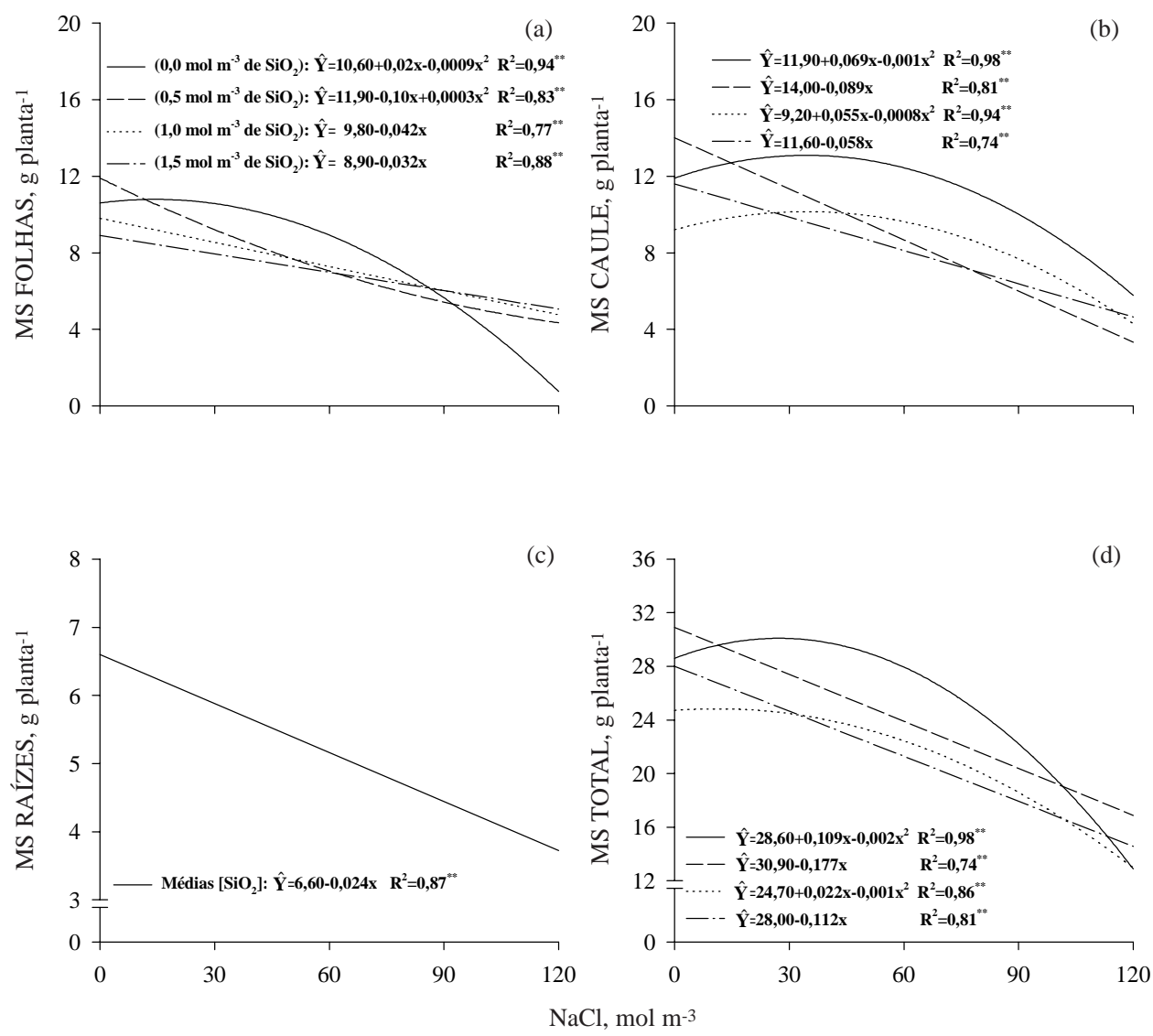

Figura 5. Acúmulo de matéria seca pelas folhas (a), caule (b), raízes (c) e acúmulo total (d), considerando a concentração de $\mathrm{NaCl}$ e $\mathrm{SiO}_{2}$. 


\section{CONCLUSÕES}

1. O Si aumentou os teores foliares de $\mathrm{K}$ e Ca e reduziu os de $\mathrm{Na}$ e $\mathrm{Cl}$. As relações $\mathrm{Na} / \mathrm{K}, \mathrm{Na} / \mathrm{Ca}$ e $\mathrm{Na} / \mathrm{Mg}$ revelaram seu potencial em preservar a integridade da membrana celular das plantas de moringa submetidas ao estresse salino.

2. Apesar dos teores crescentes de Si nas fol has, não se observou redução nos efeitos depressivos do $\mathrm{NaCl}$ sobre a produção de matéria seca das fol has, caule e raízes das plantas de moringa.

\section{LITE RATURA CITADA}

AL-KARAKI, G.N. Barley response to salt stress at varied levels of phosphorus. J. Plant Nutr., 20:1635-1643, 1997.

AWAD, A.S.; EDWARDS, D.G. \& CAMPBELL, L.C. Phosphorus enhancement of salt tolerance of tomato. Crop Sci., 30:123$128,1990$.

BENZIL, M.L.; HASEGAWA, P.M.; HANDA, A.K. \& BRESSAN, R.A. Adaptation of tobacco cells to $\mathrm{NaCl}$. Plant Physiol., 79:118-125, 1989.

BERNSTEIN, L. \& AYRES, D. Salt tolerance of five varieties of carrots. Proc. Am. Soc. Hortic. Sci., 61:360-366, 1953.

BOTELLA, M.A.; CERDÁ, A. \& LIPS, S.H. Kinetics of $\mathrm{NO}_{3}^{-}$and $\mathrm{NH}^{+}{ }_{4}$ uptake by wheat seedlings. Effect of salinity and nitrogen source. J. Plant Physiol., 144:53-57, 1994.

BOTELLA, M.A.; MARTINÉZ, V.; NIEVES, M. \& CERDÁ, A. Effect of alinity on growth and nitrogen uptake by wheat seedlings. J. Plant Nutr., 20:793-804, 1997.

BRADBURY, M.\&AHMAD, R. The effect of silicon on the growth of Prosopis juliflora in saline soil. Plant Soil, 125:71-74, 1990.

BREMNER, J .M. \& EDWARDS, A.P. Determination and isotope ratio analysis of different forms of nitrogen in soils. I. Apparatus and procedures for destillation and determination for ammonium. Soil Sci. Soc. Am. Proc., 29:504-507, 1965.

BROETTO, F. Efeito de estresse salino e biológico sobre o metabolismo de calos e suspensão de células de feijão (Phaseolus vulgaris L.). Piracicaba, Escola Superior de Agricultura "Luiz de Queiroz", 1995. 124p. (Tese de Doutorado)

CÁCERES, A.; FREIRE, V.; GIRON, L. M.; AVILÉS, O. \& PACHECO, G. Moringa oleifera (Moringaceae): Ethnobotanical Studies In Guatemala. E con. Bot., 45:522523, 1991.

CHEESEMAN, J.M. Mechanisms of salinity tolerance in plantas. Plant Physiol., 87:547-550, 1988.

FROMMER, W.B.; LUDEWIG, U. \& RENTSCH, D. Taking trnasgenic plants with a pinch of salt. Science, 285:12221223, 1999.
FROTA, J.N. \& TUCKER, T.C. Salt and water stresse influences nitrogen metabolism in red kidney beans. Soil Sci. Soc. Am. J., 42:743-746, 1978.

FURLANI, P.R. \& GALLO, J.R. Determinação de silício em material vegetal, pelo método colorimétrico do "Azul-demolibdênio". Bragantia, 37:5-9, 1978. (Nota, 2)

GERALDSON, C.M. Control of blonsom-end rot of tomatoes. Proc. Am. Soc. Hortic. Sci., 69:309-317, 1957.

GREENWAY, H. \& MUNNS, R. Mechanisms of salt tolerance in nonhalophytes. Ann. Rev. Plant Physiol., 31:149190, 1980.

HOAGLAND, D.R. \& ARNON, D.I. The water culture methods for growing plants without soil. California Agriculture Experimental Station, Berkley, 1950. 32p. (Circular 347)

HSIAO, T.C.;ACEVEDO, E.;FERERES, E.\& HENDERSON, D.W. Water stress, growth and osmotic adjustment. Philos. Trans. R. Soc. London Serv. Bull., 273:471-500, 1976.

J AHN, SA.A. Tradicional Indonesian and Ethiopia Recipes for TreeVegetables. Entwicklung-ländlicher Raun, 1:27-29, 1992

JEFFREY, W.D. \& IZQUIERDO, J . Frijol: fisiologia del potencial del rendimiento y la tolerancia al estrés. Santiago, FAO, 1989. 91p.

JESCKE, W.D. \& WOLF, O. Effect of $\mathrm{NaCl}$ salinity on growth, development, ion distribution, and ion translocation in castor bean (Ricinus comunis L.). J. Plant Physiol., 32:4553, 1988.

KRAMER, D.; LAUCHLI, A.; YEO, A.R. \& GULLASCH, J. Transfer cells in roots of Phaseolus coccineus: Ultrastructure and possible function exclusion of sodiun from the shoot. Ann. Bot., 41:1031-1041, 1977.

LIAO, C.F.H. Devards alloy method for total nitrogen determination. Soil Sci. Am. J., 45:852-855, 1981.

LIANG, Y.; SHEN, Q.; SHEN, Z. \& MA, T. Effects of silicon on salinity tolerance of two barley cultivars. J. Plant Nutr., 19:173-183, 1996.

LYNCH, J. \& LAUCHLI, A. Salt stress disturbs the calcium nutrition of barley (Hordeum vul gare) N ew Phytol., 99:345354, 1995.

MALAVOLTA, E.; VITTI, G.C. \& OLIVEIRA, S.A. Avaliação do estado nutricional das plantas. Princípios e aplicações. 2. ed. Piracicaba, POTAFOS, 1997. 319p.

MARSCHNER, H. Mineral nutrition of higher plants. 2.ed. London, Academic Press, 1995. p.889.

MATOH, T.; KAIRUSMEE, P. \& TAKAHASHI, E. Salt-induced demage to rice plants and alternation effect on silicate. Soil Sci. Plant Nutr., 32:295-304, 1986.

MIRANDA, J.R.P. \& CARVALHO, J.G.C. Acúmulo de matéria seca, macronutrientes e $\mathrm{Na}$ em plantas de (Moringa ol eifera) submetidas ao estresse salino. FERTBIO 98, Caxambu, 1998, Resumos. Caxambu, 1998. p.56.

MIRANDA, J .R.P. Silício e cloreto de sódio na nutrição mineral e produção de matéria seca de plantas de cajueiro anãoprecoce (Anacardium accidentaleL.) e de moringa (M oringa oleifera Lam.) Lavras, Universidade Federal de Lavras, 2000. p.186. (Tese de Doutorado) 
MORTON, J.F. The Horseradish Tree, Moringa pterygosperma (Moringaceae) - A Boon To Arid Lands. Econ. Bot., 45:318333, 1991.

PEREZ-ALFOCEA, F.; ESTAN, M.T.; SANTA CRUZ, A. \& BOLARIN, M.C. Effect of salinity on nutrient, total nitrogen, solube protein and free amino acid levels in tomato plants. J. Hortic. Sci., 68:1021-1027, 1993.

PETER, K.V. Drumstick. A mulpipurpose Vegetale. Ind. Hortic., 1:17-18, 1979.

REGGIANI, R.; BOZO, S. \& BERTANI, A. The effect of salinity on early seedling growth of seeds of three wheat (Triticum aestivum L.) cultivars. Can. J. Plant Sci., 75:175-177, 1995.
SAUR, E.; LAMBROT, C.; LOUSTAU, D.; ROTIVAL, N. \& TRICHET, P. Growth and uptake of mineral elements in response to sodium chloride of three provenances of maritime pine. J. Plant Nutr., 18:243-256, 1995.

YEO, A.R. Salinity resistence: physiologies and prices. Physiol. Plant., 58:214-222, 1983.

YEO, A.R.; CAPORNA, S.J .M. \& FLOWERS, T.J . The effect of salinity upon photosynthesis in rice (Oryza sativa): gas exchange by individual leaves in relation to their salt content. J. Exp. Bot., 36:1240-1248, 1985.

ZIMMERMAN, U. Physics of turgor and osmorregulation. Ann. Rev. Plant Physiol., 29:121-148, 1978. 
J .R.P. MIRANDA et al. 